\title{
Production of cinnamyl alcohol glycosides by biotransformation in roseroot callus cells
}

\author{
Iman Mirmazloum ${ }^{1,2}\left(\mathbb{D} \cdot\right.$ Attila Kiss $^{2} \cdot$ Márta Ladányi $^{3} \cdot$ Zsuzsanna György $^{4}$
}

Received: 16 May 2019 / Accepted: 29 July 2019 / Published online: 1 August 2019

(c) The Author(s) 2019

\begin{abstract}
Cinnamyl alcohol glycosides (CAGs) are the signature compounds of all roseroot preparations. The rapid growth in the market of roseroot-based products and the increasing demand for its raw material is causing serious threat for wild growing roseroot populations worldwide, which promotes the extensive studies to come up with alternative production resources. In this study the biotransformation of several precursors for the production of CAGs (rosin, rosavin, rosarin) in in vitro roseroot callus culture has been surveyed. Phenylalanine, trans-cinnamic acid, cinnamaldehyde and cinnamyl alcohol; the proposed precursors of CAGs, were added one by one to roseroot liquid callus cultures in $2 \mathrm{mM}$ concentration. Samples were harvested and analysed by HPLC after 24, 48 and $96 \mathrm{~h}$ along with controls. All of the studied compounds, except phenylalanine, promoted the formation of CAGs. It was found that the closer the position of precursors to the final product in the biosynthesis pathway the more effective was the biotransformation into the final CAGs, both in terms of time and final product quantities. Addition of $2 \mathrm{mM}$ trans-cinnamic acid and Cinnamyl alcohol resulted in 80-fold increase after $24 \mathrm{~h}$ and 130 -fold increase after $96 \mathrm{~h}$ in callus samples, respectively. Rosin was the only compound which was released into the medium from the callus cells during the experiment and only in the cinnamyl alcohol-fed callus cells. These results revealed the potential of the precursors of the cinnamyl alcohol glycosides to be subject of biotransformation by roseroot callus cultures in order to produce the pharmaceutically important compounds.
\end{abstract}

\section{Key message}

Biotransformation of precursors in Rhodiola rosea callus culture has proved to be a feasible approach to sustainably enhance the content of active constituents in vitro.

Keywords Cinnamyl alcohol glycosides $\cdot$ Precursor feeding $\cdot$ Cinnamyl alcohol $\cdot$ Rosavin $\cdot$ Rosarian $\cdot$ Rosin

Communicated by Konstantin V. Kiselev.

Electronic supplementary material The online version of this article (https://doi.org/10.1007/s11240-019-01659-7) contains supplementary material, which is available to authorized users.

Iman Mirmazloum

mirmazloum.iman@kertk.szie.hu

1 Department of Plant Physiology and Plant Biochemistry, Szent István University, Ménesi Str 44, 1118 Budapest, Hungary

2 Food Science Innovation Centre, Kaposvár University, Kaposvár, Hungary

3 Department of Biometrics and Agricultural Informatics, Szent István University, Budapest, Hungary

4 Department of Genetics and Plant Breeding, Szent István University, Budapest, Hungary

$\begin{array}{ll}\text { Abbreviations } \\ \text { 2iP } & \mathrm{N}^{6} \text {-(2-Isopentenyl) adenosine } \\ \text { BAP } & \text { 6-BenzylAminoPurine } \\ \text { CAGs } & \text { Cinnamyl alcohol glycosides } \\ \text { IAA } & \text { Indole-3-Acetic Acid } \\ \text { NAA } & \alpha \text {-NaphthaleneAcetic Acid } \\ \text { PAL } & \text { Phenylalanine ammonia lyase } \\ \text { Phe } & \text { Phenylalanine } \\ \text { TCA } & \text { trans-cinnamic acid }\end{array}$

\section{Introduction}

Rhodiola rosea L. (roseroot or golden root) is one of the well-known medicinal plants with adaptogennic properties. It is a perennial herbaceous plant, a dioecious member of the Crassulaceae plant family. $R$. rosea is distinguished from 
the other species of the genus and honoured for its outstanding pharmacological importance and use (Tasheva and Kosturkova 2012). Cinnamyl alcohol glycosides (CAGs) (rosin, rosarin and rosavin) along with salidroside are the most important constituents of $R$. rosea extracts (Zapesochnaya and Kurkin 1982). A wide range of experimental research demonstrated its adaptogenic effects, including anti-fatigue, antidepressant, anxiolytic, stimulating effect on central nervous system and many others (Panossian et al. 2010; Marchev et al. 2016).

The market demand for roseroot raw material is growing faster than the natural resources can supply for (GrechBaran et al. 2015). The endangered status of $R$. rosea and the attractive market, which is rapidly growing led to more intensive research in domestication, cultivation, and even in biotechnological techniques such as in vitro cultures (Galambosi 2014). Collection from natural sites has several drawbacks including the possible adulteration with other species (Booker et al. 2015) and the difficulty of standardizing the raw materials, since the collected roots and rhizomes are from plants of different ages (Kolodziej and Sugier 2013; Weglarz et al. 2008). The cultivation of roseroot also does not ensure the standard quality of raw materials due to the high variability in the content of the active metabolites in plants of different origins which needs a long term monitoring and investigation on the content of active ingredients to be produced from different genotypes under different cultivation conditions (Linh et al. 2000; Ganzera et al. 2001; Peschel et al. 2012; Altantsetseg et al. 2007). Different domestication efforts showed some variability in phytochemical profile of roseroot plants with different genetic background (Aiello et al. 2013; Adamczak et al. 2014). Although some efforts have been done on the cultivation of this species, but profitability of the cultivation is still questionable due to the required long-term investment, as the cultivation should last at least for 5 years (Galambosi 2006; Weglarz et al. 2008). The combination of the above factors resulted in a huge diversity in the quality and characteristics of available roseroot materials for manufacturers and ultimately the final consumers.

The possibility of producing the pharmaceutically important substances of roseroot in in vitro cultures started in the 90's (Furmanowa et al. 1995). However, it turned out that callus cells do not accumulate the desired secondary metabolites under the in vitro condition (Kurkin et al. 1991; Furmanowa et al. 1999b; György et al. 2004). Several in vitro studies aimed to stimulate and enhance the production of roseroot active metabolites among which the precursor feeding seems to be the most efficient approach (Xu et al. 1998; Shi et al. 2013; Grech-Baran et al. 2015).

CAGs are the products of phenylpropanoid metabolism, derived from L-phenylalanine (György 2006). Their biosynthesis starts from L-phenylalanine (Fig. 1), which is
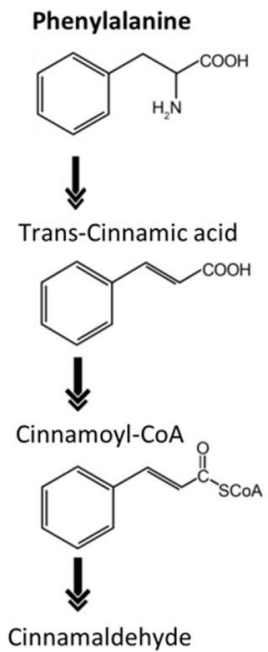

Cinnamaldehyde
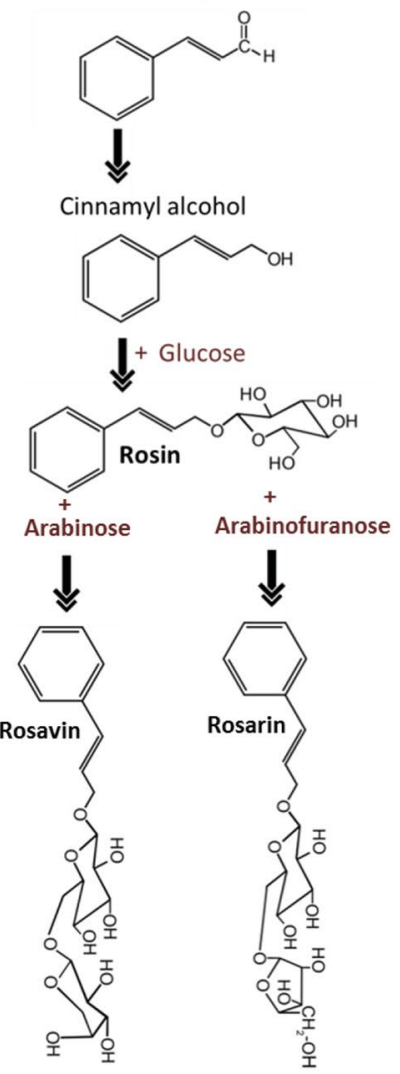

Fig. 1 Proposed biosynthetic pathway of roseroot's cinnamyl alcohol glycosides

converted into cinnamic acid by PAL. From cinnamic acid, cinnamyl-CoA ester is formed by the activity of hydroxycinnamate: CoA ligase. This CoA ester is further reduced to cinnamaldehyde by cinnamyl-CoA reductase. The cinnamaldehyde is further reduced by cinnamyl alcohol dehydrogenase to produce cinnamyl alcohol (György 2006; Grech-Baran et al. 2015). The enzymes that take part in the formation of the glycosides of cinnamyl alcohol are not yet described. The binding of glucose to cinnamyl alcohol 
would result in rosin formation, which is the simplest cinnamyl alcohol glycoside. Finally, rosavin and rosarin can be formed from rosin by the connection of an arabinopyranose and arabinofuranose unit, respectively.

So far, the precursor feeding experiments aiming for enhanced production of CAGs only focused on the biotransformation of cinnamyl alcohol. Furmanowa et al. (1999a) were the first who successfully applied trans-cinnamyl alcohol $(2.5 \mathrm{mM})$ to the medium of roseroot callus cells in suspension culture where $90 \%$ of the given precursor was converted into a number of unidentified products, among which only rosavin was identified. Later, György et al. (2004) applied different concentrations of cinnamyl alcohol $(0.05-5 \mathrm{mM})$ in callus culture and proposed the optimum $2 \mathrm{mM}$ concentration with no adverse effect on plant cells growth with significant improvement in transformation of given substrate to rosin and rosavin. In another study the biotransformation rate of cinnamyl alcohol was significantly increased (doubled) when glucose was supplemented into the culture medium (György et al. 2005). Similar results were reported by Krajewska-Patan et al. (2007) in the same biotransformation reaction on solid medium. In addition, a de novo biosynthesis of rosin was achieved where biosynthetic pathway of cinnamyl alcohol was constructed in Escherichia coli, suggesting the role of two UGT genes in glucosylation of cinnamyl alcohol (Zhou et al. 2017).

The aim of this study was to explore the roseroot callus cells' biotransformation capacity upon supplementation of not only cinnamyl alcohol, but all the proposed intermediate compounds of the CAGs biosynthetic pathway for enhancing the production of rosin, rosavin and rosarian in vitro.

\section{Materials and methods}

\section{Plant materials and in vitro culture}

Seeds of Rhodiola rosea $\mathrm{L}$. were kindly provided by the Botanical Garden of University of Oulu (Finland). To establish the in vitro culture (Fig. 2), $R$. rosea seeds were surface sterilized by immersion in $70 \%$ ethanol for 3 min followed by submerging in 50\% sodium hypochlorite for $4 \mathrm{~min}$ and then rinsed four times in sterile distilled water. For germination, half-strength MS medium (Murashige and Skoog
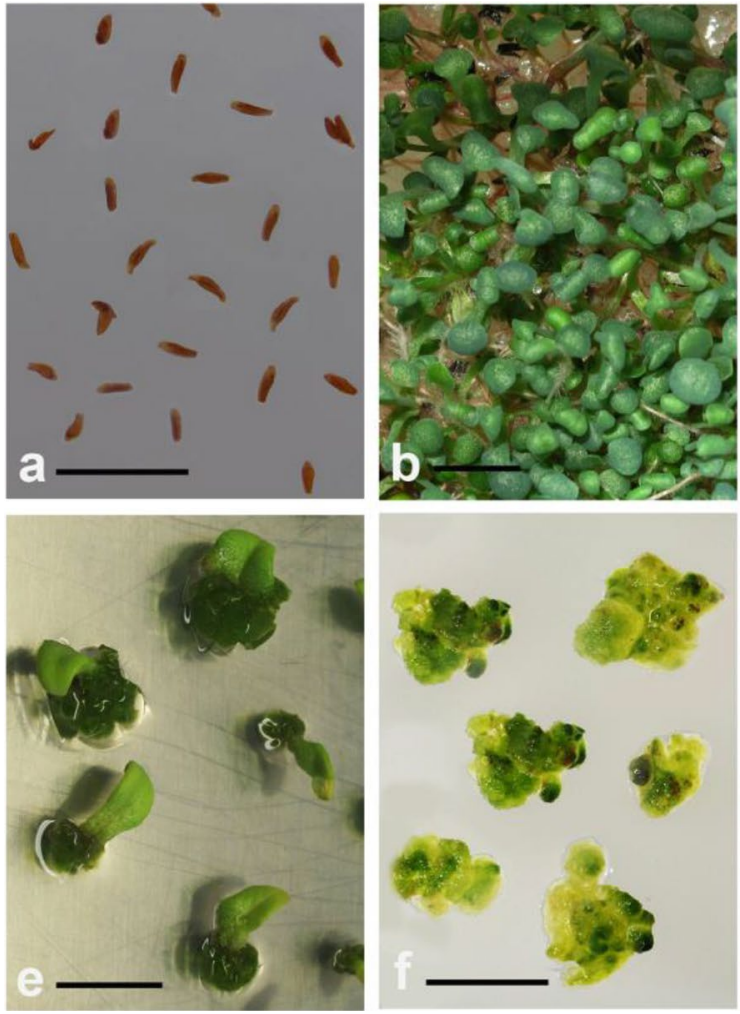

Fig. 2 Roseroot callus induction and liquid culture for biotransformation of precursors of CAGs biosynthesis $($ Bars $=1 \mathrm{~cm}$ ). a Disinfected roseroot seeds on MS medium. b 4 weeks-old roseroot seedlings. c 4-months-old roseroot seedlings used for callus induction from the leaves. d Leaves of roseroot on callus-inducing solid MS medium enriched $30 \mathrm{~g} / \mathrm{L}$ sucrose, gelled with $4.5 \mathrm{~g} / \mathrm{L}$ agar and supplemented
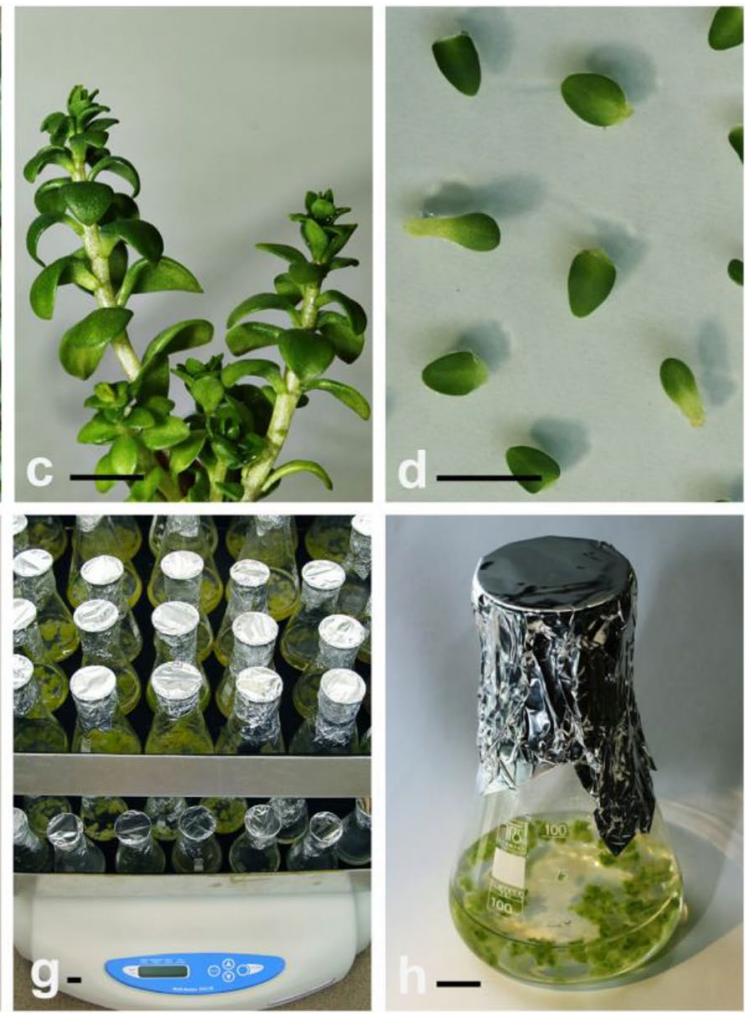

with $3 \mathrm{mg} / \mathrm{L} 2 \mathrm{iP}$ and $0.3 \mathrm{mg} / \mathrm{L}$ IAA phytohormones in glass Petri dishes. e Roseroot callus formation after 6 weeks. $\mathbf{f}$ Sub-culturing the vigorous roseroot callus on the fresh medium of the same composition. $\mathbf{g}$, h Roseroot liquid callus culture for biotransformation of precursors 
1962) containing $30 \mathrm{~g} / \mathrm{L}$ sucrose were prepared and solidified with $4.5 \mathrm{~g} / \mathrm{L}$ agar. The $\mathrm{pH}$ was adjusted to 5.8 before sterilizing the medium by autoclaving at $121{ }^{\circ} \mathrm{C}$ for $20 \mathrm{~min}$. Under aseptic condition, $25 \mathrm{~mL}$ of medium was distributed into glass jars and 40 sterilized seeds were sown in each jar. Seeds were germinated and grown at $22 \pm 2{ }^{\circ} \mathrm{C}$ under a $16 \mathrm{~h}$ photoperiod with a photosynthetic photon flux density (PPFD) of $60 \mu \mathrm{mol} / \mathrm{m}^{2} / \mathrm{s}$ at culture level, provided by coolwhite fluorescent lamps. After seed germination, the seedlings were sub-cultured on the same medium after 6 weeks intervals.

For callus induction, three individual seedlings were selected based on good vigour 16 weeks after germination. Leaves of 4-6 $\mathrm{mm}$ in diameter were cut from the seedlings and scratched at the edges by using a sharp sterile scalpel blade. The leaves were put on the surface of fresh MS medium enriched with $30 \mathrm{~g} / \mathrm{L}$ sucrose, gelled with $4.5 \mathrm{~g} / \mathrm{L}$ agar and supplemented with $3 \mathrm{mg} / \mathrm{L} 2 \mathrm{iP}$ and $0.3 \mathrm{mg} / \mathrm{L} \mathrm{IAA}$ phytohormones (Mirmazloum et al. 2014) in glass Petri dishes for callus induction. Callus formation occurred under the same light and temperature conditions for 6 weeks. The liquid cultures for biotransformation experiments were established in liquid MS medium containing $30 \mathrm{~g} / \mathrm{L}$ sucrose supplemented with $1 \mathrm{mg} / \mathrm{L}$ NAA and $0.5 \mathrm{mg} / \mathrm{L} \mathrm{BAP.} \mathrm{Erlen-}$ meyer flasks containing roseroot calli were kept on an orbital shaker at $120 \mathrm{rpm}$ in $16 \mathrm{~h}$ photoperiod. The maintenance of the calli was insured by sub-culturing on the same medium until around $500 \mathrm{~g}$ of green homogenous roseroot callus was produced to conduct the biotransformation experiment.

\section{Precursor feeding and biotransformation}

The potential precursor compounds of cinnamyl alcohol glycosides biosynthesis pathway (Mirmazloum and György 2012) namely, phenylalanine, trans-cinnamic acid, and cinnamyl alcohol (Sigma-Aldrich, Germany) were dissolved in water while the cinnamaldehyde were dissolved in ethanol and water $\left(0.132 \mathrm{~g} / 1 \mathrm{ml} \mathrm{EtOH}+249 \mathrm{~mL} \mathrm{H}_{2} \mathrm{O}\right)$, sequentially. Stock solutions of $4 \mathrm{mM}$ were prepared from all precursors in sterile distilled water, which were filter sterilized using $0.22 \mu \mathrm{m}$ pore size, Millex GP filter unit from Millipore before the application. The $\mathrm{pH}$ was adjusted to 5.8 using $\mathrm{HCl}$ and $\mathrm{NaOH}$. The final concentration of each precursor compounds in liquid culture was $2 \mathrm{mM}$ as suggested by György (2006). About eight grams of fresh calli were washed with distilled water; surface dried, measured for exact fresh weight aseptically and placed into $50 \mathrm{~mL}$ Erlenmeyer flasks. Sixteen $\mathrm{ml}$ of each biotransformation liquid medium $(2 \mathrm{mM}$ of each precursor, MS medium, $30 \mathrm{~g} / \mathrm{L}$ sucrose) was added to each flask in triplicate. Two sets of control (liquid culture without any added precursor compounds and biotransformation medium containing each pressure without roseroot callus) were also prepared in triplicate in this experiment.
The flasks were kept on an orbital shaker at $120 \mathrm{rpm}$ in the light room. Callus samples were harvested from each biotransformation flasks and controls after 24, 48 and $96 \mathrm{~h}$ of culture initiation. Medium samples $(5 \mathrm{~mL})$ were also collected from each corresponding flasks. Five $\mathrm{ml}$ of each culture medium was also taken at the start of the experiment and stored at $-20{ }^{\circ} \mathrm{C}$ for HPLC analysis as original reference. The harvested samples $(\sim 4 \mathrm{~g} \mathrm{FW})$ were washed with distilled water (three times) and surface dried on paper towel and finally dried in an oven overnight at $45^{\circ} \mathrm{C}$. The dried calli and medium samples were analysed by HPLC.

\section{Extraction and HPLC analysis}

From each dried callus sample $0.5 \mathrm{~g}$ was grounded for extraction. To prepare the medium samples, $5 \mathrm{~g}$ of liquid medium was filtered and $4 \mathrm{~g}$ of filtrate was dried at $45^{\circ} \mathrm{C}$ in a vacuum rotary evaporator. Extraction was performed in $1.5 \mathrm{~mL}$ of $70 \%$ methanol in an ultrasonic bath for $1 \mathrm{~h}$ at room temperature. A clear extract was obtained after centrifugation (8000 rpm; $10 \mathrm{~min}$ ) and used for HPLC analysis. The same procedure was applied for preparing the medium samples when the remaining film of dried medium was dissolved in $2 \mathrm{~mL}$ of $70 \%$ methanol in $50 \mathrm{~mL}$ round bottom flasks and transferred to Eppendorf tubes for centrifugation. The chromatography was performed using Waters 1525 binary pump, with 717 autosampler and 2998 PDA detector on a reversed phase Thermo Hypersil ODS $250 \times 4.65 \mu \mathrm{m}$ column at $40{ }^{\circ} \mathrm{C}$ with a neutral mobile phase (purified water and acetonitrile) gradient system at a flow rate of $1.0 \mathrm{~mL} /$ min and UV detection at 205, 222, 254 and $280 \mathrm{~nm}$ simultaneously (Supplementary Table S1). Injection volume was $10 \mu \mathrm{L}$ and the run time was $31 \mathrm{~min}$. Peaks were identified by comparison of retention time (Supplementary Table S1) and spectral data with adequate parameters of standards $(R$. rosea Standards Kit, ChromaDex, USA; phenylalanine, tyrosine, tyrosol, trans-cinnamic acid, cinnamaldehyde and cinnamyl alcohol from Sigma-Aldrich, Germany). Quantification was performed based on the peak area and the content of the determined compounds was calculated as $\mathrm{mg} / 100 \mathrm{~g}$ of roseroot callus dry weight.

\section{Statistical analysis}

Two-way multivariate ANOVA (MANOVA) was used to compare the treatment and elapsed time effect. Having significant overall result, univariate follow-up ANOVA was run with Bonferroni's Type I error correction. Normality of the error terms was accepted according to the skewness and kurtosis of the error terms. Since the homogeneity of variances 
was violated, Games-Howell's post hoc test was applied for pairwise comparisons regarding the significant factor(s).

\section{Results}

We conducted in vitro experiments in which the proposed precursors of cinnamyl alcohol glycosides (phenylalanine, trans-cinnamic acid, cinnamaldehyde and cinnamyl alcohol) were supplied to $R$. rosea callus cells cultured in liquid medium for biotransformation reaction analysis.

The precursors were dissolved in the culture medium in a final concentration of $2 \mathrm{mM}$ and the callus samples were harvested 24, 48 and $96 \mathrm{~h}$ after the feeding. The HPLC analysis showed no changes in the content of applied compounds in the control medium (without the roseroot cells) during $96 \mathrm{~h}$ under the same culture condition indicating that no spontaneous changes have been occurred. Therefore, we assumed that all changes in the content of the given compounds were the results of interaction with roseroot callus cells. In the presented figures, charts without lower axis (Figs. 4 and 5) are representing the compounds of which no trace amount was detectable in the culture medium.

\section{Biotransformation of phenylalanine}

Phenylalanine, the first compound in the proposed pathway showed no contribution in formation of CAGs after $96 \mathrm{~h}$. The addition of Phe resulted in its significantly higher content in the treated samples when comparted with the controls. Even though the Phe content was decreased from the medium (Fig. 3 lower axis) but its content remained constant in the harvested callus samples during this experiment
Fig. 3 Effect of $2 \mathrm{mM}$ phenylalanine treatment on phenylalanine, tyrosine and tyrosol content in roseroot callus culture. No significant time effect was detected on control and on treated samples $(p>0.05)$. In comparison of control versus treated samples, significantly higher values are marked with star. Error bars represent standard deviation of the mean among three biological replicates. *The detected content from $2 \mathrm{mM}$ phenylalanine after $24 \mathrm{~h}$ considered as $100 \%$

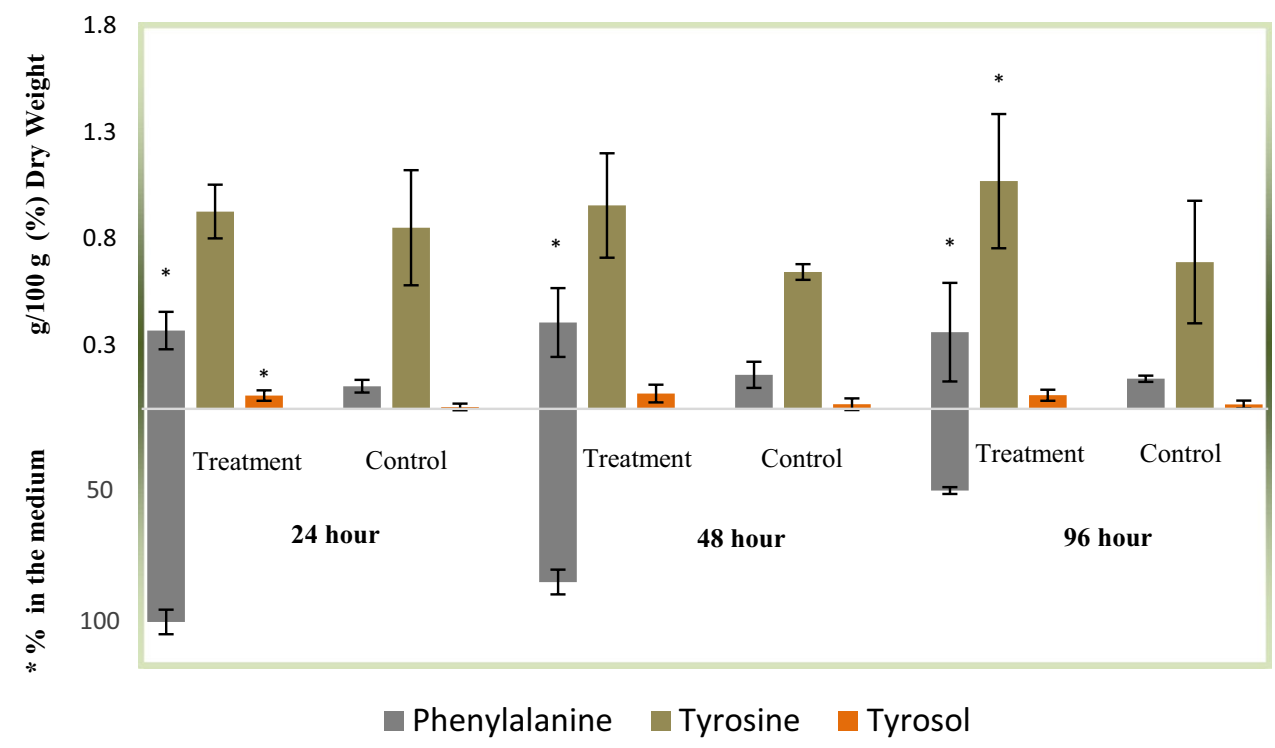

Fig. 4 Effect of $2 \mathrm{mM}$ transcinnamic acid treatment on trans-cinnamic acid, rosin, rosarin and rosavin content in roseroot callus culture. Different letters are for significantly different time effect on treated samples (Games-Howell, $p<0.05)$. No significant time effect was detected on control samples $(p>0.05)$. In comparison of control versus treated samples, significantly higher values are marked with star. Error bars represent standard deviation of the mean among three biological replicates

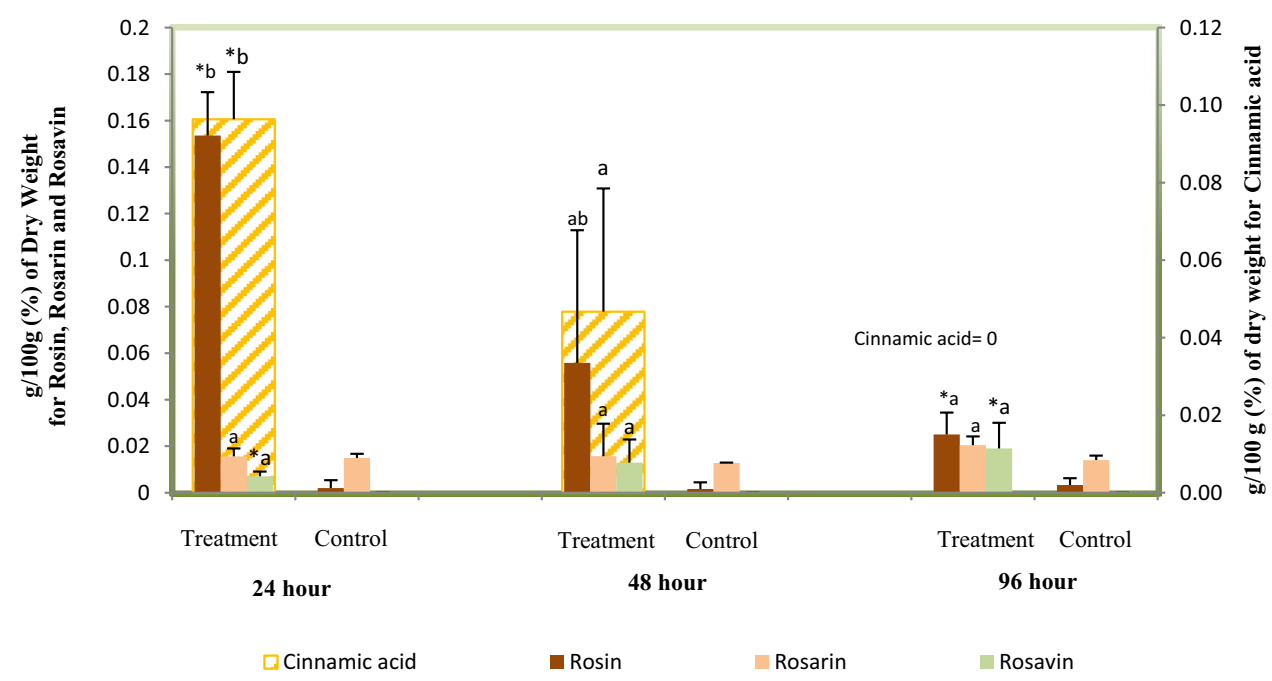


Fig. 5 Effect of $2 \mathrm{mM}$ cinnamaldehyde treatment on cinnamaldehyde, cinnamyl alcohol, rosin, rosarin and rosavin content in roseroot callus culture. Different letters are for significantly different time effect on treated samples (Games-Howell, $p<0.05$ ). No significant time effect was detected on control samples $(p>0.05)$. In comparison of control versus treated samples, significantly higher values are marked with star. Error bars represent standard deviation of the mean among three biological replicates

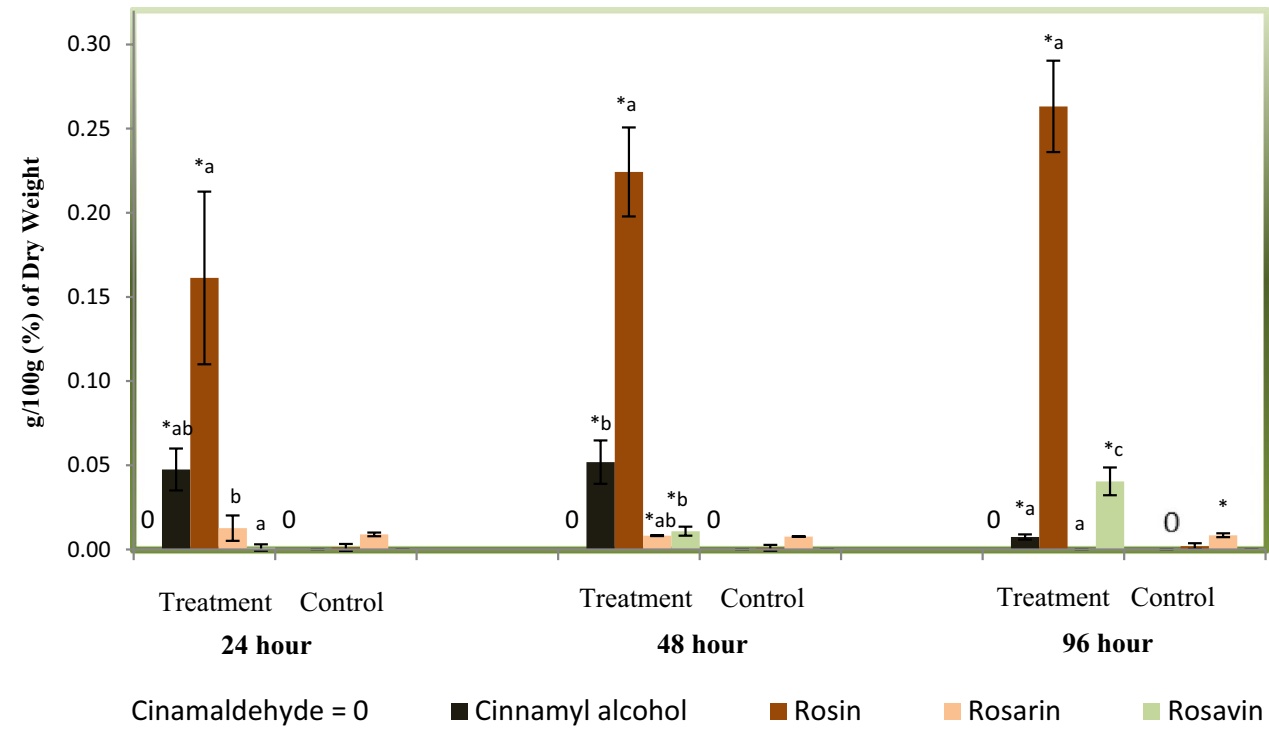

(Fig. 3 upper axis). The overall treatment effect was only significant for Phe that could be up taken by the plant cells, but has not been used in biotransformation reactions (time: Wilks $\lambda=0.570 ; \mathrm{p}=0.664$; treatment: Wilks $\lambda=0.053$; $\mathrm{p}<0.001)$, the follow-up univariate ANOVA is significant $(\mathrm{F}(1 ; 12)=134.302 ; \mathrm{p}<0.001$; CAGs: $\mathrm{F}(1 ; 12)<4.1$; $\mathrm{p}>0.05)$. Surprisingly, Phe significantly interacted with tyrosine increasing its content after $96 \mathrm{~h}$ of roseroot callus culture when compared to untreated controls.

\section{Biotransformation of trans-cinnamic acid}

Applying trans-cinnamic acid increased the overall content of CAGs (Fig. 4). No TCA was remained in the liquid medium after $24 \mathrm{~h}$. Its utilization by roseroot calli were confirmed when high amount of TCA was accumulated in the cells after $24 \mathrm{~h}$ compering to the control samples with none. The TCA content gradually decreased to 0 after $96 \mathrm{~h}$, reflecting its consumability in biotransformation reactions. The rosin content increased from 1.1 to $92 \mathrm{mg} / 100 \mathrm{~g} \mathrm{DW}$ (more than 80 -fold) after $24 \mathrm{~h}$ of feeding. The rosin content gradually decreased in the following days, still being significantly higher than the controls. As the TCA and rosin content declined in the cells, the rosavin content increased inversely. The content of rosavin, which was not detected in the controls at all, showed a gradual increase during the experiment. Rosarin was detected in both the treated and control samples where its content did not differ significantly after TCA feeding.

Two-way MANOVA resulted in significant treatment and time effect together with significant interaction (Wilks $\lambda=0.021,0.045$ and 0.044 , respectively with $\mathrm{p}<0.001)$. Follow-up univariate ANOVA with Bonferroni's Type I error correction revealed significant treatment effect in case of TCA, rosin and rosavin $(\mathrm{F}(1 ; 12)>41.7 ; \mathrm{p}<0.001)$ but insignificant for rosarin $(\mathrm{F}(1 ; 12)=1.332 ; \mathrm{p}=0.271)$. As for time effect, we detected significance result in case of TCA and $\operatorname{rosin}(\mathrm{F}(2 ; 12)=18.025 ; \mathrm{p}<0.01$ and $\mathrm{F}(2 ; 12)=10.7$; $\mathrm{p}<0.001$, respectively) while the changes were not significant for rosarin and rosavin $(\mathrm{F}(2 ; 12)=0.360 ; \mathrm{p}=0.705$ and $\mathrm{F}(2 ; 12)=1.446 ; \mathrm{p}=0.274$, respectively). According to Games-Howell's post hoc test, treated sample values of 24 and $48 \mathrm{~h}$ were significantly different in case of TCA and rosin.

\section{Biotransformation of cinnamaldehyde}

Cinnamaldehyde was found neither in the medium nor in the cells after $24 \mathrm{~h}$ of treatment (Fig. 5) meaning that it was up taken by the cells and completely utilized in biotransformation reactions. Cinnamyl alcohol which was absent in control samples was found in the treated cells when its content declined by the end of the experiment presumably being used in further metabolic reactions. The rosin content was gradually increased from 161 to $263 \mathrm{mg} / 100 \mathrm{~g}$ DW during $96 \mathrm{~h}$ in the treated samples during $96 \mathrm{~h}$ with a 130-fold increase when compared with untreated controls ( $\simeq 2 \mathrm{mg} / 100 \mathrm{~g} \mathrm{DW}$ ) as a result of $2 \mathrm{mM}$ cinnamaldehyde feeding. The rosavin content also showed a gradual and significant increase as a result of cinnamaldehyde treatment with a reverse correlation to the declined quantity of cinnamyl alcohol. The rosarin content also changed significantly after $48 \mathrm{~h}$ in callus culture. Two-way MANOVA resulted in significant treatment and time effect together with significant interaction (Wilks $\lambda=0.004,0.014$ and 0.017 , respectively with $\mathrm{p}<0.001)$. Follow-up univariate ANOVA with Bonferroni's Type I error correction revealed significant treatment effect in case of cinnamyl alcohol, rosin and rosavin 
$(\mathrm{F}(1 ; 12)>105 ; \mathrm{p}<0.001)$ which was insignificant for rosa$\operatorname{rin}(\mathrm{F}(1 ; 12)=0.885 ; \mathrm{p}=0.365)$. The time effect was significant for all the four measured compounds in treated samples $(\mathrm{F}(2 ; 12)>5.9 ; \mathrm{p}<0.05)$ according to Games-Howell's post hoc test.

\section{Biotransformation of cinnamyl alcohol}

Cinnamyl alcohol is the aglycone of all CAGs, significantly effective to increase both the rosin and rosavin content when supplied in callus culture, with no effect on rosarin content (Fig. 6). Rosin was the only compound, which was released into the medium from the callus cells during all experiments and only from the cinnamyl alcohol fed samples (Fig. 6 lower axis). Analysis of the medium samples revealed that the cinnamyl alcohol was gradually up taken from the medium by the cells when its content decreased from 100 to $2 \mathrm{mg} / 100 \mathrm{~g} \mathrm{DW}$ after $24 \mathrm{~h}$ (Fig. 6 lower axis). A correlation was found between the decrease in rosin and increase in rosavin content during the experiment as expected according to the proposed CAGs pathway. The two-way MANOVA showed significant effect of treatment and time and also their interaction (Wilks $\lambda=0.006,0.104$ and 0.103 , respectively with $\mathrm{p}<0.001)$. Follow-up univariate ANOVA resulted in significant treatment effect for all the four measured compounds $(\mathrm{F}(1 ; 12)>15 ; \mathrm{p}<0.01)$. The time effect was significant for rosin and rosavin content $(\mathrm{F}(2 ; 12)=6.514 ; \mathrm{p}<0.05$ and $\mathrm{F}(2 ; 12)=10.427 ; \mathrm{p}<0.01$, respectively) while it was not significant for rosarin $(\mathrm{F}(2 ; 12)=2.228 ; \mathrm{p}=0.150)$. GamesHowell's post hoc test detected significant differences among treated sample values of rosavin between 24 and $48 \mathrm{~h}$ and of rosin between 48 and $96 \mathrm{~h}$. The results of precursor feeding experiments verified the biotransformation potential of in vitro $R$. rosea callus cells indicating production inducibility of CAGs suing precursors feeding method.

\section{Discussion}

In in vitro plant cultures, plant secondary metabolites are produced under eco-friendly environment and according to GMP standards, thus ultimately providing continuous production of BioSafe, bioactive and homogenous natural products. This approach eliminates the influence of environmental and seasonal factors and does not threaten the natural populations of rare and endangered plant species.

Roseroot grows very slowly in its natural habitats where the accumulation of adequate levels of its active metabolites takes 5 to 7 years (Furmanowa et al. 1999b; Galambosi 2006). There limited number of reports concerning roseroot secondary metabolite production in callus and cell suspension cultures (György 2015) mainly due to the fact that in vitro cultures were not proven to be efficient enough to compete with the wild growing plants in terms of the active constituents and content. Almost all reports admitted the lack or only traces of secondary metabolites content of roseroot in in vitro trials (György et al. 2004; György and Hohtola 2009; Martin et al. 2010).

We have shown that all precursors of cinnamyl alcohol glycosides except phenylalanine could be efficiently utilized in biotransformation reactions by in vitro grown roseroot callus cells. We have found that the more efficient biotransformation (considering the time and quantity) was
Fig. 6 Effect of $2 \mathrm{mM}$ cinnamyl alcohol treatment on cinnamyl alcohol, rosin, rosarin and rosavin content in roseroot callus culture. Different letters are for significantly different time effect on treated samples (Games-Howell, $p<0.05$ ). No significant time effect was detected on control samples $(p>0.05)$. In comparison of control vs treated samples, significantly higher values are marked with star. Error bars represent standard deviation of the mean among three biological replicates. ${ }^{\dagger}$ The detected content from $2 \mathrm{mM}$ cinnamyl alcohol after $24 \mathrm{~h}$ considered as $100 \%$

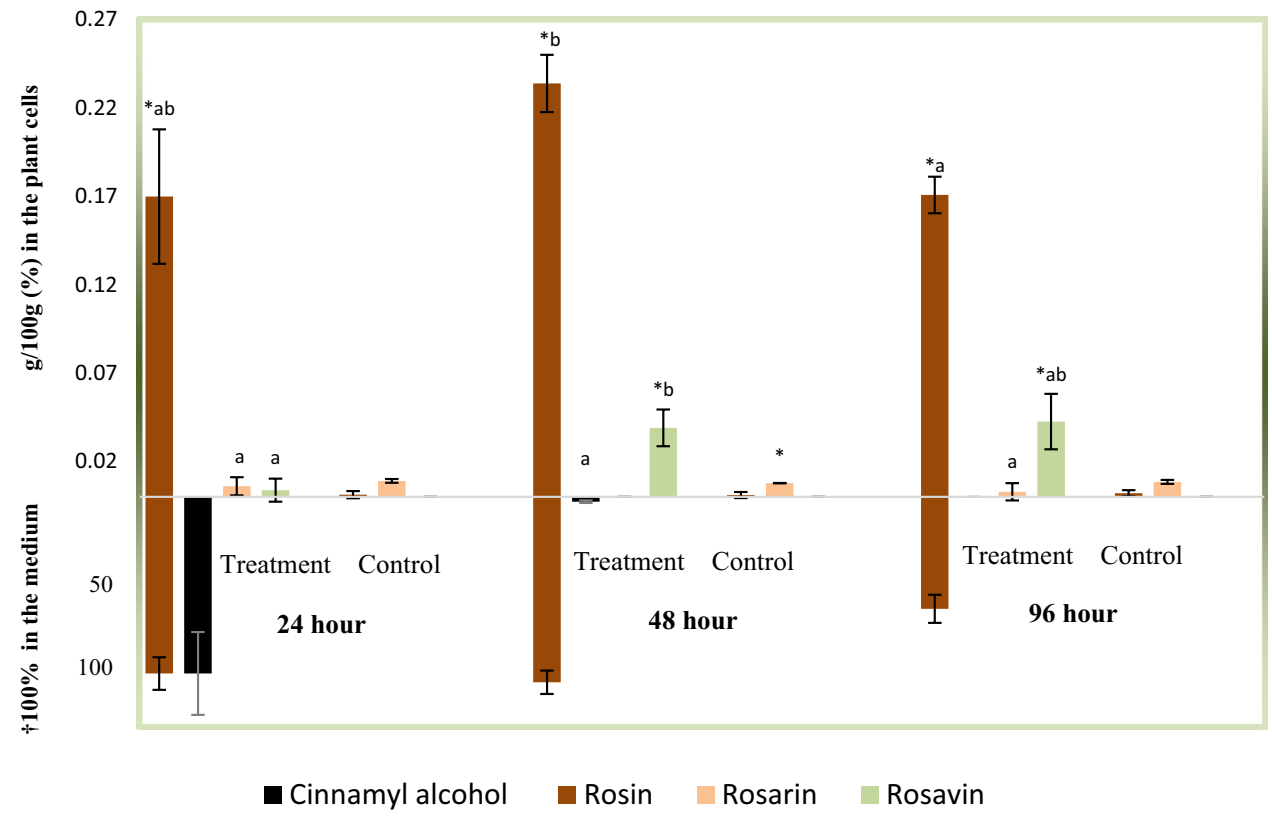


taking place from the less upstream precursors in the pathway of desired glycosides biosynthesis.

Biotransformation of TCA resulted in the accumulation of rosin and rosavin, without detecting the precursors in between. Following the feeding of cinnamaldehyde all upstream compounds of CAGs biosynthesis have been detected except rosarin. We could observe a real time stepwise conversion of studied molecules in their biosynthesis root. The content of cinnamyl alcohol increased after culture initiation following it's accumulation in the treated roseroot cells; it's content decline during the 96 $\mathrm{h}$ period; and its conversion to the upstream compounds realized by increase in accumulated rosin and rosavin content. The feeding of cinnamyl alcohol and cinnamaldehyde had approximately the same effect in the callus cells with regard to CAGs overall content. The cinnamaldehyde supplementation resulted in excessive rosin production by the cells to the extent that it has been released into the medium which can be an important indicator for optimization of applied precursor's quantity.

Cinnamyl alcohol is the only compound from the CAGs biosynthesis route, which has been applied in biotransformation studies. Supplementation of cinnamyl alcohol at 2 and $2.5 \mathrm{mM}$ enhanced the rosin and rosavin content to 0.72 and $1.01 \%$ DW, respectively when compared to zero in controls (György et al. 2004; Furmanowa et al. 1999a). Surprisingly the content of rosarin was not affected by the feeding of any of the precursors that might be discussed considering its specific structure. Rosin is the simpler glycoside of cinnamyl alcohol containing a glucose molecule whereas in case of rosavin and rosarin an extra arabinose (rosavin) or arabinofuranose (rosarin) is present (Mirmazloum and György 2012). Even though the aglycone (in this case rosin) was present in the cells the availability of arabinofuranose might be a limiting factor for rosarin formation. Although the interconversion of arabinopyranose and arabinofuranose which is essential for cell wall establishment in plant development performed by mutases of reversibly glycosylated proteins gene family has been reported (De Pino et al. 2007) but their activity under the in vitro condition may not be enough to provide sufficient quantity of required arabinofuranose for rosarian biosynthesis. Production of rosarin might be enhanced by applying exogenous arabinofuranose to be directly used in biotransformation reaction.

Our results gave a broader picture of the biosynthesis of the CAGs. The timing of the uptake of the precursors from the medium and their conversions to other molecules are more clarified after our applied biotransformation method. This timing information is an interesting indicator of the biotransformation capacity, which can be optimized to its highest possible level. The overall findings from this experiment showed a remarkable potential in this technique, which can be used in many different fields from molecular studies to bioreactor production of such desired phytochemicals.

Acknowledgements Open access funding provided by Szent István University (SZIE). This research was supported by NKFIH-FK128793 project and the Higher Education Institutional Excellence Program (20430-3/2018/FEKUTSTRAT) awarded by the Ministry of Human Capacities within the framework of plant breeding and plant protection researches of Szent István University. Dr. Gabriella Szalai (Centre for Agricultural Research, Hungarian Academy of Sciences) is acknowledged for performing the HPLC analysis.

\section{Compliance with ethical standards}

Conflict of interest The authors declare that they have no conflict of interest.

Open Access This article is distributed under the terms of the Creative Commons Attribution 4.0 International License (http://creativeco mmons.org/licenses/by/4.0/), which permits unrestricted use, distribution, and reproduction in any medium, provided you give appropriate credit to the original author(s) and the source, provide a link to the Creative Commons license, and indicate if changes were made.

\section{References}

Adamczak A, Gryszczynska A, Buchwald W (2014) Biometric and phytochemical variability of roseroot (Rhodiola rosea L.) from field cultivation. Herba Pol 60(1):7-17

Aiello N, Bontempo R, Vender C, Innocenti G, Dall'Acqua A (2013) Morphological and qualitative characteristics of Rhodiola rosea L., wild populations of Trentino, Italy. Z Arznei- Gewurzpfla 18(1):41-45

Altantsetseg K, Przybyl JL, Weglarz Z, Geszprych A (2007) Content of biologically active compounds in roseroot (rhodiola $\mathrm{Sp}$.) raw material of different derivation. Herba Pol 53(4):1-7

Booker A, Jalil B, Frommenwiler D, Reich E, Zhai L, Kulic Z, Heinrich M (2015) The authenticity and quality of Rhodiola rosea products. Phytomedicine 23(7):754-762

De Pino V, Borán M, Norambuena L, González M, Reyes F, Orellana A, Moreno S (2007) Complex formation regulates the glycosylation of the reversibly glycosylated polypeptide. Planta 226:335-345

Furmanowa M, Oledzka H, Michalska M, Sokolnicka I, Radomska D (1995) Rhodiola rosea L. (Roseroot): In vitro regeneration and the biological activity of roots. In: Bajaj YPS (ed) Medicinal and aromatic plants VIII, biotechnology in agriculture and forestry, Springer, Berlin, pp 412-426

Furmanowa M, Hartwich M, Alfermann AW (1999a) Salidroside as a product of biotransformation by Rhodiola rosea cell suspension cultures. Book of abstracts: 2000 years of natural products research. Amsterdam, p. 152

Furmanowa M, Hartwich M, Alfermann AW, Koźmiński W, Olejnik M (1999b) Rosavin as a product of glycosylation by Rhodiola rosea (roseroot) cell cultures. Plant Cell Tissue Organ Cult 56(2): $105-110$

Galambosi B (2006) Demand and availability of Rhodiola rosea L. row material. In: Bogers R, Cracer L, Lange D (eds) Medicinal and aromatic plants. Springer, Netherlands, pp 223-236

Galambosi B (2014) Cultivation of Rhodiola rosea in Europe. In: Cuerrier A, Ampong-Nyarko K (eds) Rhodiola rosea- traditional herbal medicine for modern time. CRC Press, Florida, pp 87-125 
Ganzera M, Yayla Y, Khan IA (2001) Analysis of the marker compounds of Rhodiola rosea L. (golden root) by reversed phase high performance liquid chromatography. Chem Pharm Bull 49(4):465-467

Grech-Baran M, Syklowska-Baranek K, Pietrosiuk A (2015) Biotechnological approaches to enhance salidroside, rosin and its derivatives production in selected Rhodiola spp. in vitro cultures. Phytochem Rev 14:657-674

György Z (2006) Glycoside production by in vitro Rhodiola rosea cultures. Acta Universitatis Ouluensis C Technica 244. Oulu University Press, Oulu

György Z (2015) Biotechnology of Rhodiola rosea. In: Cuerrier A, Ampong-Nyarko K (eds) Rhodiola rosea-traditional herbal medicine for modern time. CRC Press, Florida, pp 173-187

György Z, Hohtola A (2009) Production of cinnamyl glycosides in compact callus aggregate cultures of Rhodiola rosea through biotransformation of cinnamyl alcohol. In: Mohn J, Saxena PK (eds) Protocols for in vitro cultures and secondary metabolite analysis of aromatic and medicinal plants. The Humana Press Inc., New York, pp 305-312

György Z, Tolonen A, Pakonen M, Neubauer P, Hohtola A (2004) Enhancement of the production of cinnamyl glycosides in CCA cultures of Rhodiola rosea through biotransformation of cinnamyl alcohol. Plant Sci 166(1):229-236

György Z, Tolonen A, Neubauer P, Hohtola A (2005) Enhanced biotransformation capacity of Rhodiola rosea callus cultures for glycosid production. Plant Cell Tissue Organ Cult 83:129-135

Kolodziej B, Sugier D (2013) Influence of plants age on the chemical composition of roseroot Rhodiola rosea L.). Acta Sci Pol-Hortoru 12(3):147-160

Krajewska-Patan A, Dreger M, Lowicka A, Górska-Paukszta M, Mscisz A, Mielcarek S, Baraniak M, Buchwald W, Furmanowa M, Mrozikiewicz PM (2007) Chemical investigations of biotransformed Rhodiola rosea callus tissue. Herba Pol 53(4):77-87

Kurkin VA, Zapesochnaya GG, Dubichev AG, Vorontsov ED, Aleksandrova IV, Panova RV (1991) Phenylpropanoids of callus culture of Rhodiola rosea. Chem Nat Compd 27(4):419-425

Linh PT, Kim YH, Hong SP, Jian JJ, Kang JS (2000) Quantitative determination of salidroside and tyrosol from the underground part of Rhodiola rosea by high performance liquid chromatography. Arch Pharma Res 23(4):349-352

Marchev AS, Dinkova-Kostova AT, György Z, Mirmazloum I, Aneva IY, Georgiev MI (2016) Rhodiola rosea L.: from golden root to green cell factories. Phytochem Rev 15(4):515-536

Martin J, Pomahacova B, Dusek J, Duskova J (2010) In vitro culture establishment of Schizandra chinensis (Turz.) Baill. and Rhodiola rosea L., two adaptogenic compounds producing plants. J Phytol 11:80-87

Mirmazloum I, György Z (2012) Review of the molecular genetics in higher plants towards salidroside and cinnamyl alcohol glycosides biosynthesis in Rhodiola rosea L. Acta Alim 41(1):133-146

Mirmazloum I, Forgács I, Zok A, Pedryc A, György Z (2014) Transgenic callus culture establishment, a tool for metabolic engineering of Rhodiola rosea L. Acta Sci Pol Hortoru 13(4):95-106

Murashige T, Skoog F (1962) A revised medium for rapid growth and bioassays with tobacco tissue cultures. Physiol Plant 15(3):473-497

Panossian A, Wikman G, Sarris J (2010) Rosenroot (Rhodiola rosea): traditional use, chemical composition, pharmacology and clinical efficacy. Phytomedicine 17:481-493

Peschel W, Prieto JM, Karkour C, Williamson EM (2012) Effect of provenance, plant part and processing on extract profiles from cultivated European Rhodiola rosea L. for medicinal use. Phytochemistry 86:92-102

Shi L, Wang C, Zhou X, Zhang Y, Liu Y, Ma C (2013) Production of salidroside and tyrosol in cell suspension cultures of Rhodiola crenulata. Plant Cell, Tissue Organ Cult 114(3):295-303

Tasheva K, Kosturkova G (2012) The role of biotechnology for conservation and biologically active substances production of Rhodiola rosea —endangered medicinal species. Sci World J 274942:1-13

Weglarz Z, Przybyl JL, Geszprych A (2008) Roseroot (Rhodiola rosea L.): effect of internal and external factors on accumulation of biologically active compounds. In: Ramawat KG, Mérillon JM (eds) Bioactive Molecules and Medicinal Plants. Springer, Berlin, pp 297-315

Xu JF, Ying PQ, Han AM, Su ZG (1998) Enhanced salidroside production in liquid-cultivated compact callus aggregates of Rhodiola sachalinensis: manipulation of plant growth regulators and sucrose. Plant Cell, Tissue Organ Cult 55(1):53-58

Zapesochnaya GG, Kurkin VA (1982) Glycosides of cinnamyl alcohol from the rhizomes of Rhodiola rosea. Chem Nat Compd 18(6):685-688

Zhou W, Bi H, Zhuang Y, He Q, Yin H, Liu T, Ma Y (2017) Production of cinnamyl alcohol glucoside from glucose in Escherichia coli. J Agric Food Chem 65:2129-2135

Publisher's Note Springer Nature remains neutral with regard to jurisdictional claims in published maps and institutional affiliations. 